\title{
Full field residual stress determination using hole-drilling and electronic speckle pattern interferometry (ESPI) with phase unwrapping method
}

\author{
J.-N. Aoh ${ }^{1, a}$ and B.-L. Lyu ${ }^{1}$ \\ ${ }^{1}$ National Chung Cheng University, Department of Mechanical Engineering, Minhsiung, Chiayi, 621 \\ Taiwan
}

\begin{abstract}
The hole-drilling strain gauge technique has become a standard method in measuring residual stresses [1]. Moiré interferometry combining hole-drilling method opens additional opportunity for full-field residual stress measurement using optical interferometry [2]. The optical moiré method has a non-contact feature comparing with strain gauge method. Yet Moiré interferometry suffers a drawback in its complicated grating preparation on one hand and it is difficult to be applied to work piece with complicated geometry on the other hand. Electronic speckle pattern interferometry (ESPI) provides information about the displacement field of a surface and it can be conveniently used on asreceived surfaces without special surface preparation and can be applied to work piece with complicated geometry that may be unsuitable for applying strain gauge or gratings. Studies on combining ESPI with hole-drilling show that is feasible to obtain reasonable residual stress values [3, 4]. The purpose of this study was to demonstrate the detail of hole-drilling technique combining ESPI with phase unwrapping method to reveal the full field stress distribution and to measure the associated stress field on a thin specimen exerted by a uni-axial load. This study also demonstrates the noise reduction achieved by Gaussian low pass filter and a successful phase unwrapping resulted from five-step phase shifting and cellular automata method.
\end{abstract}

Figure 1 shows the experimental setup of the ESPI system and the hole-drilling system. The light from a laser source is split into two beams. One split beam emerges from a PZT-stage to provide stepwise phase shifting and it further interferes with the other split image beam on the specimen surface to produce speckle patterns onto the CCD camera. By recording the speckle images of stepwise phase shifting before and after hole-drilling, the fringe patterns at each step can be obtained. Through a uniaxial loading fixture loads with different stress magnitudes in the range between 50 $\mathrm{MPa}$ and $150 \mathrm{MPa}$ were applied to produce different degrees of loading and the associated fringe patterns. A five-step phase shifting was used in this work. Continuous phase distribution was achieved using phase unwrapping method.

Gaussian low pass filter was applied and a suitable cut-off frequency was selected to effectively reduce the noise of patterns especially in the vicinity of the hole and to obtain a continuous phase map with closed contours. The continuous phase maps were reconstructed with higher quality and lower noise by applying the Schmitt's five-step phase shifting algorithm and cellular automata (CA) phase unwrapping method. Special caution should be put on the phase unwrapping around the hole.

\footnotetext{
a e-mail : imejna@ccu.edu.tw
} 


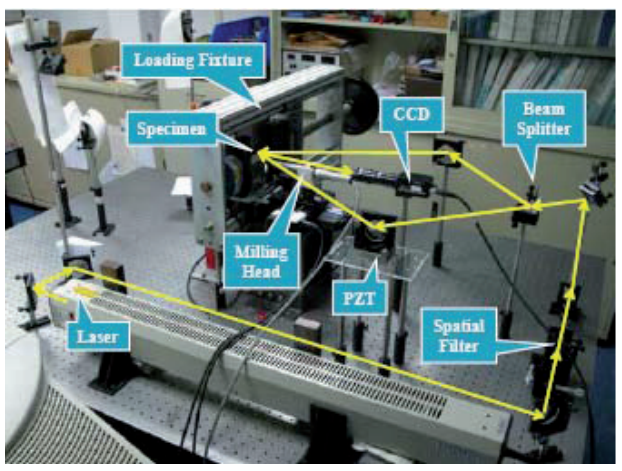

Fig. 1. The experimental setup of the ESPI system and the hole-drilling system

Reducing the image area considered in the CA computation effectively reduced the CA computation time with stress values still in the range with satisfactory accuracy. Theoretical fringe patterns at each phase shifting step and at different stress magnitudes were constructed to compare with the experimental ones, Fig. 2. The relationship between displacement, stress and the number of fringes and the change of fringe patterns was analyzed to validate the feasibility of the ESPI combining hole-drilling method and to verify the accuracy of the stress results. Error analysis shows that the error between theoretical results and experimental data is in general less than $20 \%$
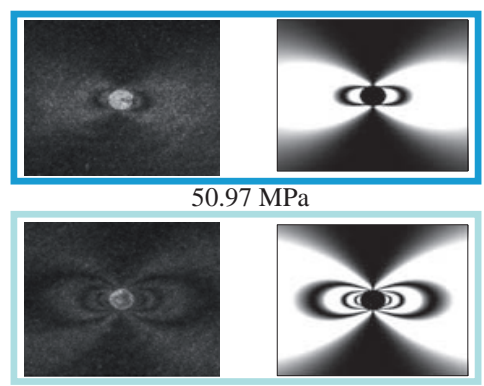

98.29 MPa

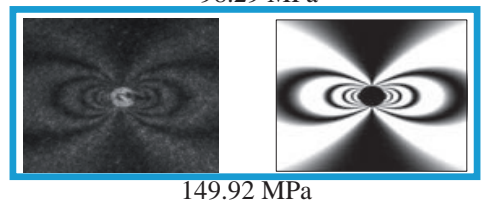

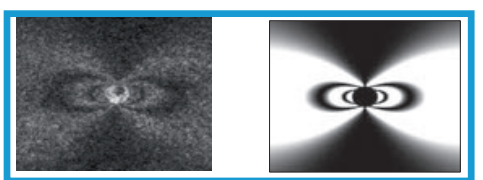

$74.03 \mathrm{MPa}$

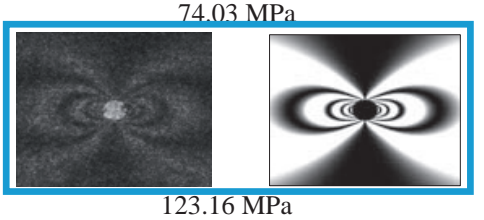

Fig. 2. Comparison between theoretical and experimental fringe patterns.

\section{References}

1. ASTM E837-01, "Standard Test Method for Determining Residual Stresses by the HoleDrilling Strain-Gage Method." American Society for Testing and Materials, West

Conshohocken, PA. (2001).

2. M. Ya, H. Miao, X. Zhang, Opt. \& Laser in Engrg., 44, (2006).

3. F. V. Diaz, G.H. Kaufmann, G.E. Galizzi, Opt. \& Laser in Engrg., 33, (2000).

4. M. R. Viotti, Opt. \& Laser in Engrg., 41, (2002). 